\title{
Grain Growth in Thin Films with a Fibre Texture Studied by Phase-Field Simulations and Mean Field Modelling
}

\section{Citation}

Moelans, N., Frans A. Spaepen, and P. Wollants. 2010. Grain growth in thin films with a fiber texture studied by phase-field simulations and mean field modeling. Philosophical Magazine 90(1-4): 501-523.

\section{Published Version}

doi:10.1080/14786430902998129

\section{Permanent link}

http://nrs.harvard.edu/urn-3:HUL.InstRepos:4262331

\section{Terms of Use}

This article was downloaded from Harvard University's DASH repository, and is made available under the terms and conditions applicable to Open Access Policy Articles, as set forth at http:// nrs.harvard.edu/urn-3:HUL.InstRepos:dash.current.terms-of-use\#OAP

\section{Share Your Story}

The Harvard community has made this article openly available.

Please share how this access benefits you. Submit a story.

\section{Accessibility}


Vol. 00, No. 00, Month 0000, 1-34

\title{
RESEARCH ARTICLE
}

\section{Grain growth in thin films with a fiber texture studied by phase-field simulations and mean field modeling}

\author{
N. Moelans ${ }^{\mathrm{a}, \mathrm{b} *}$, F. Spaepen ${ }^{\mathrm{c}}$ and P. Wollants ${ }^{\mathrm{a}}$ \\ ${ }^{a}$ K.U.Leuven, Dept. Metallurgy and Materials Engineering, Kasteelpark Arenberg 44, bus \\ 2450, Leuven, B-3001, Belgium; ${ }^{\mathrm{b}}$ Lawrence Livermore National Laboratory, Condensed \\ Matter $\&$ Materials Division, 7000 East Avenue, Livermore, CA 94551; ${ }^{\mathrm{C}}$ Harvard \\ University, School of Engineering and Applied Sciences, 29 Oxford Street, Cambridge,
} MA 02138, USA;

The evolution of fiber textured structures is simulated in 2 dimensions using a generalized phase field model assuming two forms for the misorientation dependence of the grain boundary energy. In each case, a steady-state regime is reached after a finite amount of grain growth, where the number and length weighted misorientation distribution functions (MDF) are constant in time, and the mean grain area $A$ as a function of time $t$ follows a power growth law $A-A_{0}=k t^{n}$ with $n$ close to 1 and $A_{0}$ the initial mean grain area. The final shape of the MDF and value of the prefactor $k$ in the power growth law clearly correlate with the misorientation dependence of the grain boundary energy. From a quantitative point of view, the fraction of special boundaries obtained in simulations is quite sensitive to the number of possible discrete orientations. Furthermore, a mean field approach is worked out to predict the growth exponent for systems with nonuniform grain boundary energy. The conclusions from the mean field approach are consistent with the simulation results.

Keywords: Phase Field Model; Anisotropy; Grain Growth; Polycrystalline Thin Film;

\footnotetext{
*Corresponding author. Email: nele.moelans@mtm.kuleuven.be
}

ISSN: 1478-6435 print/ISSN 1478-6443 online

(c) 0000 Taylor \& Francis

DOI: 10.1080/1478643YYxxxxxxxx

http://www.informaworld.com 


\section{Introduction}

Many thin films have a columnar grain structure in which all crystals have nearly identical orientation in the axial direction (the direction perpendicular to the film), but random radial orientation (in the plane of the film) [1]. The symmetry is for example introduced by a preferential nucleation or growth of certain grain orientations due to anisotropy in the surface energy or film-subrate interfacial energy. Many technically important characteristics of the films, such as their strength, conductivity, corrosion resistance or resistance against void formation, are correlated with characteristics of the grain structure and grain boundary network [2-5]. Moreover, the structure and properties of the grain boundaries can vary considerably, depending on the misorientation between the adjacent grains and the inclination of the boundary with respect to the crystal lattices of the grains [6-12].

Grain growth in systems with non-uniform grain boundary properties has been studied frequently, experimentally [13-16] and by mesoscale simulations [17-24]. Due to the large number of parameters, there is however no systematic insight yet in the correlations between grain boundary properties and grain structure evolution. that would allow us to predict how a given grain structure evolves in time and optimize the film properties for a particular application.

The experiments of Saylor et al. [13] and 3D simulations of Gruber et al. [17] show that the inclination dependence of the grain boundary energy affects the grain boundary plane distribution for bulk structures with a random texture. After a limited amount of grain growth, a stead-state is established with a constant grain boundary plane distribution with maxima at inclinations with low energy. Inclination dependence of the grain boundary mobility seems to have no effect on 
dependence of the mobility can induce texture formation in a 3D structure with random orientations. It however occurred at the end of simulations and is thus probably due to the restricted number of grain orientations left in the system. Holm et al. [18] performed 2D simulations of grain growth in anisotropic systems using 3D crystallography (the orientations are represented with 3 Euler angles). They consider a Read-Shockley misorientation dependence for the energy of boundaries with low misorientation [6],

$$
\sigma_{g b}(\theta)=\sigma_{m} \frac{|\theta|}{\theta_{m}}\left(1-\ln \left(\frac{|\theta|}{\theta_{m}}\right)\right)
$$

where $\theta$ represents the smallest rotation angle between the orientations of two adjacent grains and with $\theta_{m}=15,30$ and $45^{\circ}$ and $\sigma_{m}$ the energy of the boundary with misorientation $\theta_{m}$. The energy of high-angle boundaries and the mobility of all boundaries are constant. They find that structures with a random texture show grain growth behavior that is very similar to normal grain growth in isotropic materials. After a short transition period, the average grain size increases in time according to a power growth law

$$
A-A_{0}=k t^{n} \quad \text { or } \quad A=k t^{n} \text { for } t \rightarrow \infty,
$$

with $A$ the mean grain area, $k$ a kinetic constant and $n=1$. Furthermore, the normalized grain size distribution has the same shape as for normal grain growth in isotropic structures. The MDF (misorientation distribution function) is constant in time, but low-energy boundaries have a higher probability than given by the Mackenzie distribution which is obtained for isotropic materials. They explain that 
dynamic equilibrium at triple junctions namely forces the low-energy boundaries to lengthen, while the number fraction of low-energy boundaries hardly increases in their simulations. Mobility anisotropy seems not to influence the evolution of a structure with random texture. Ono et al. [27] and Hassold et al. [19] consider the effect of extra low-energy cusps in the grain boundary energy at large misorientations, as is the case for some CSL (Coincidence Site Lattice)-boundaries. Ono et al. report an increase of the length fraction for all misorientations with low energy. Hassold et al. assumes narrower cusps around the special high-angle misorientations and observes only an increase of the fraction of low-angle boundaries, probably because the possibility to form a high-angle boundary with low energy in a structure with random grain orientations (and using 3D crystallography) is very low in their systems.

Different from structures with a random texture, structures with all grain orientations near a single orientation have the tendency to strengthen this texture. The simulations of Holm et al. [18], assuming the same misorientation dependence for the grain boundary properties as described in the previous paragraph with $\theta_{m}=15^{\circ}$, but starting from a grain structure with all orientations around $\{111\}<100>$, demonstrate that the length and number fraction of the smallest misorientations increase continuously. The growth exponent $n$ in the power law equals 0.62 and the grain size distribution is weighted towards smaller grains compared to that obtained for normal grain growth in isotropic systems. Furthermore, the grain structures contain many few sided grains and stable quadruple-junctions. The average number of sides is smaller than 6. Kazaryan et al. [20] study highly textured systems with misorientation and weak inclination dependence of the grain boundary energy and mobility, but using only 1 scalar orientation variable. If grain 
aries increases seriously in time and the growth exponent $n$ is around 0.74 at an intermediate stage of the simulation, but increases continuously. The edge distribution also shifts towards grains with less than 6 sides. Mobility anisotropy alone does not change the power growth law, the MDF or the edge distribution. Different from what is observed for randomly textured systems [17], inclination dependence of the mobility leads to a continuous evolution of the grain shape and grain boundary inclination distribution, even if the grain boundary energy is uniform. When both grain boundary energy and mobility anisotropy are considered, the misorientation dependence of the mobility affects the grain growth exponent and evolution of the MDF.

Strongly textured systems that contain a few grains with random orientation are likely to undergo abnormal grain growth $[21,28,29]$ caused by anisotropy in grain boundary energy or mobility. If the fraction of randomly oriented grains is higher (12.5\%-27\%), there is no abnormal grain growth; however the fraction of randomly oriented grains increases or decreases continuously in time, depending on the relative degrees of anisotropy for grain boundary energy and mobility [22]. Structures with initially 2 or 3 strong texture components, show often a step-wise growth in which periods of fast growth and strengthening of 1 of the components are interrupted by periods of slow growth and negligible change in the fractions of the texture components $[25,30-33]$. In all these cases, the evolution is extremely sensitive to the initial fraction and spacial distribution of the different texture components and randomly oriented grains. Furthermore, both energy and mobility anisotropy can change the MDF and growth exponent $n$. The analytical mean field study of Kazaryan et al. [34] for 2D systems indeed demonstrates that, in general, anisotropy of the grain boundary mobility alone can lower the exponent $n$ 
component and for randomly textured structures, which is also seen in simulations.

In the case of a perfect fiber texture, the axi-symmetry allows us to describe the grain boundary properties as a function of a single variable $\theta$ representing the misorientation measured around the common tilt axis. Different from a fully random 3D crystallography, the possibility to form a boundary with a certain misorientation is equal for all possible misorientations. The possibility to form a low-angle boundary is accordingly larger than in randomly textured 3D structures, but smaller than in structures with a strong texture component. Grest et al. [23] and $\mathrm{Yu}$ and Essche [35] examined the evolution of systems with a Read-Shockley dependence (equation (1)) for the energy of low-angle boundaries $\left(\theta<\theta_{m}\right)$ and constant energy for high-angle boundaries $\left(\theta>\theta_{m}\right)$. To study the effect of the width of the Read-Shockley well, simulations were performed for $\theta_{m}$ between 0 and $\pi$, assuming 2-fold orientation symmetry. Grest et al. find that the growth exponent $n$ is approximately 0.84 for $\theta_{m}<\pi / 2$ and changes gradually from 0.84 for $\theta_{m}=\pi / 2$ to 0.5 for $\theta=\pi$. The length fraction of the boundaries with the lowest energy at the end of the simulations is around $10 \%$, but varies with $\theta_{m}$. The number of stable quadruple junctions is higher and the grain size distribution becomes broader for larger values of $\theta_{m} . \mathrm{Yu}$ and Essche, however, find that regardless the width of the Read-shockley well, the growth exponent equals 1. At the beginning of the simulations, there is an increase of the number of quadruple-junctions for large values of $\theta_{m}$, but in all cases this value decreases again during the simulations. The average number of sides remains close to 6 . Yu and Essche devote the different findings for both studies to differences in the Monte Carlo algorithm used for the simulations, as the system properties and size are almost the same in both studies. A growth exponent $n=0.84$, as obtained by Grest et al., is obviously 
too low for $\theta_{m} \rightarrow$ U. Moreover, a Read-Shockley dependence with $\theta_{m}>15$ is

rather artificial. Saito and Enomoto [36] performed grain growth simulations for fiber textures using a grain boundary energy misorientation dependence based on experimental measurements for $\mathrm{Cu}$ with several low-energy cusps. At the end of the simulations, the fraction of low-energy boundaries is approximately $20 \%$ and the growth exponent equals 1 . The grain size distribution broadens during anisotropic grain growth. Upmanyu et al. [24] compare simulation results obtained with a Potts model and a phase field model. The misorientation dependence of grain boundary energy and mobility are derived from molecular dynamics simulations $\left(\theta>10^{\circ}\right)$ and theoretical models $\left(\theta<10^{\circ}\right)$. For low misorientations a Read Shockley dependence (1) with $\theta_{m}=10^{\circ}$ is assumed. The misorientation dependence shows six-fold symmetry. If grain boundary energy anisotropy alone is considered the growth exponent $n$ equals 0.93 if only a Read-Shockley well is considered (Potts simulations), and 0.86 (Potts simulations) or 0.65 (phase field simulations) if extra cusps at large misorientations are also considered. If both energy and mobility are anisotropic, the growth exponent is 0.69 (Potts simulations) or 0.77 (phase field simulation). The fraction of boundaries with lower energy increases in time, also for those with large misorientation. There are stable higher order junctions in the structures and the average number of edges is lower than 6. Mobility anisotropy alone does not affect the growth exponent $n$. Frost et al. [37] report the evolution towards a steady-state regime with $n=1$ and a constant grain boundary character distribution for thin films with columnar grains and random grain orientations in the plane of the film.

Previous simulations for fiber textured systems thus indicate that although the grain orientations in the plane of the film are random, the MDF does not remain random when grain boundary energy is anisotropic. It is not clear whether the 
structures, or whether a steady-state is established after a finite amount of grain growth, as for randomly textured structures. Furthermore, there is a large scatter on the values obtained for the growth exponent $n$ and there is no systematic insight in how the number, depth, width and position of low-energy or low-mobility cusps influence the results.

In this paper, the evolution of fiber textured systems with misorientation dependent energy is studied by means of phase field simulations assuming two different spectra for the grain boundary energy. It is discussed how the mean grain size and MDF evolve in time. Moreover, a more general mean field analysis is performed to predict the growth exponent for systems with non-uniform grain boundary properties. Since the purpose is to study the effect of the misorientation dependence of the grain boundary energy on the evolution of a fiber texture, effects from grain boundary grooving at the film surface and mismatches between the film and the substrate are not considered.

\section{Phase-Field Simulation Method}

Several simulation techniques have been proposed for the simulation of grain growth, such as Monte Carlo, vertex, front tracking, phase-field and cellular automata [11]. The phase-field method has proven to be a versatile technique for simulating microstructure evolution; it has been applied for solidification, solid state phase transitions and coarsening [39-42]. One advantage of the phase-field technique for the current application is that the simulation results are less influenced by the anisotropic nature of the discretization grid because grain boundaries are represented as diffuse regions with a finite width. 
In this section the phase field model used for the simulations is briefly discussed. A more extensive explanation is given in [43, 44]. Different grain orientations are represented by a large set of independent and nonconcerved field variables $\eta_{1}(\mathbf{r}, t), \eta_{2}(\mathbf{r}, t), \ldots, \eta_{i}(\mathbf{r}, t), \ldots \eta_{p}(\mathbf{r}, t)$, with $p$ the number of possible grain orientations. The total free energy of the system $F$ is give by the functional

$$
F=\int_{V}\left[m f_{0}\left(\eta_{1}, \ldots, \eta_{p}\right)+\frac{\kappa_{\theta}}{2} \sum_{i=1}^{p}\left(\nabla \eta_{i}\right)^{2}\right] \mathrm{d} V
$$

with

$$
\begin{aligned}
f_{0}\left(\eta_{1}, \ldots, \eta_{p}\right) & =\sum_{i=1}^{p}\left(\frac{\eta_{i}^{4}}{4}-\frac{\eta_{i}^{2}}{2}\right) \\
& +\gamma_{\theta} \sum_{i=1}^{p} \sum_{j>i}^{p} \eta_{i}^{2} \eta_{j}^{2}+\frac{1}{4}
\end{aligned}
$$

The homogeneous free energy $f_{0}$ has localized minima at $\left(\eta_{1}, \ldots, \eta_{p}\right)=$ $(1,0, \ldots, 0),(0,1,0, \ldots, 0), \ldots,(0, \ldots, 0,1)$, where $f_{0}=0$, representing the different grain orientations. To introduce the misorientation dependence of the grain boundary energy, the parameters $\kappa_{\theta}$ and $\gamma_{\theta}$ are formulated as a function of the local values of the field variables:

$$
\begin{gathered}
\kappa_{\theta}=\frac{\sum_{i=1}^{p} \sum_{j>i}^{p} \kappa_{i, j} \eta_{i}^{2} \eta_{j}^{2}}{\sum_{i=1}^{p} \sum_{j>i}^{p} \eta_{i}^{2} \eta_{j}^{2}} \\
\gamma_{\theta}=\frac{\sum_{i=1}^{p} \sum_{j>i}^{p} \gamma_{i, j} \eta_{i}^{2} \eta_{j}^{2}}{\sum_{i=1}^{p} \sum_{j>i}^{p} \eta_{i}^{2} \eta_{j}^{2}}
\end{gathered}
$$


tations $i$ and $j$, only $\eta_{i}$ and $\eta_{j}$ are different from 0 , it can easily be verified that $\kappa_{\theta}=\kappa_{i, j}, \gamma_{\theta}=\gamma_{i, j}$ at the boundary between grains $i$ and $j$. The grain boundary energy can accordingly be specified individually for all $p-1$ possible discrete misorientations.

The spatial and temporal evolution of the field variables is calculated from the equations

$$
\frac{\partial \eta_{i}(\mathbf{r}, t)}{\partial t}=-L\left[\eta_{i}^{3}-\eta_{i}+2 \eta_{i} \sum_{j \neq i} \gamma_{i, j} \eta_{j}^{2}-\kappa_{\theta} \nabla^{2} \eta_{i}\right]
$$

for $\eta_{i}=\eta_{1} \ldots \eta_{p}$, with $L$ a kinetic model parameter related to the grain boundary mobility. These equations are obtained from the free energy functional (3) using a variational approach, but requiring that the local values of the model parameters $\kappa_{\theta}$ and $\gamma_{\theta}$ are fixed for a given grain configuration (the $\eta$-dependence in expressions 5.) Since relaxations in grain boundary structure are typically on the atomic scale for metals, we can assume that they are much faster than the mesoscale grain boundary movement and thus consider grain boundary properties as fixed for a given misorientation.

The following expressions have been derived for the grain boundary energy $\sigma_{i, j}$ and mobility $\mu_{i, j}$ of a grain boundary between grains $i$ and $j$

$$
\begin{aligned}
& \sigma_{i, j}=g\left(\gamma_{i, j}\right) \sqrt{\kappa_{i, j} m} \\
& \mu_{i, j}=\frac{L}{g\left(\gamma_{i, j}\right)} \sqrt{\frac{\kappa_{i, j}}{m}}
\end{aligned}
$$




$$
g\left(\gamma_{i, j}\right)=\int_{0}^{1} \sqrt{f_{0}\left(\eta_{i}, \eta_{j}\left(\eta_{i}\right)\right)} \sqrt{1+\left(\frac{\mathrm{d} \eta_{j}\left(\eta_{i}\right)}{\mathrm{d} \eta_{i}}\right)^{2}} \mathrm{~d} \eta_{i}
$$

The width $\ell_{i, j}$ of the profiles of the field variables across the interface between two grains (the distance over which the field variables change their values) is defined based on the maximum gradients of the order parameter fields,

$$
\begin{aligned}
\ell_{i, j} & =\frac{1}{\left|\left(\mathrm{~d} \eta_{i} / \mathrm{dx}\right)_{\max }\right|}=\frac{1}{\left|\left(\mathrm{~d} \eta_{j} / \mathrm{dx}\right)_{\max }\right|} \\
& =\sqrt{\frac{\kappa_{i, j}}{m f_{0, \text { interf }}\left(\gamma_{i, j}\right)}}
\end{aligned}
$$

with $f_{0, \text { interf }}$ the value of $f_{0}$ at the middle of the interface where the 2 fields intersect. In this way the width of the diffuse grain boundaries can be used as a parameter in criteria for the numerical stability and accuracy of the simulations. Usually it is most efficient to give all boundaries equal width. The functions $g(\gamma)$ and $f_{0 \text {,interf }}(\gamma)$ were evaluated numerically for a wide range of $\gamma$-values [44]. It was also verified by means of numerical simulations that relations (7)-(10) are valid for $\ell_{i, j} / R<1 / 5$, with $R$ the radius of curvature of the boundary.

\section{2. $\quad$ System properties and parameter choice}

Two forms of misorientation dependence of the grain boundary energy are considered. Both have a Read-Shockley dependence of the form (1) with $\theta_{m}=15^{\circ}$ and $\sigma_{m}=0.25 \mathrm{~J} / \mathrm{m}^{2}$ for misorientations smaller than $15^{\circ}$. For the first type (Type I), all large misorientations $\left(\theta>15^{\circ}\right)$ have the same energy $\sigma_{m}=0.25 \mathrm{~J} / \mathrm{m}^{2}$. For the 


$$
\sigma_{g b}(\theta)=\left(\sigma_{m}-\sigma_{l}\right) \frac{|\theta-37.5|}{\theta_{m}^{\prime}}\left(1-\ln \left(\frac{|\theta-37.5|}{\theta_{m}^{\prime}}\right)\right)+\sigma_{l}
$$

for $27.5^{\circ} \leq \theta \leq 45^{\circ}$ and with $\sigma_{l}=0.1$ the energy of the special high-angle boundary at $37,5^{\circ}$ and $\theta_{m}^{\prime}=10^{\circ}$ a measure for the width of the cusp. The remaining misorientation angles have an energy $\sigma_{g b}=0.25 \mathrm{~J} / \mathrm{m}^{2}$. A fourfold symmetry is assumed in each case. The orientations within one quadrant are discretized with an interspacing $\Delta \theta$ equal to $1.5^{\circ}$ or $3^{\circ}$ and the discrete orientations are assigned to respectively 60 or 30 field variables. The misorientation between grains with orientations $i$ and $j$ is calculated as

$$
\theta=\Delta \theta(|j-i|) \quad \text { if } \quad|j-i| \leq p / 2
$$

or

$$
\theta=-90^{\circ}+\Delta \theta^{\circ}(|j-i|) \quad \text { if } \quad|j-i| \geq p / 2
$$

with $p$ the number of discrete orientations. Two neighboring grains with a misorientation smaller than $\Delta \theta$ are treated as one grain. The 2 energy spectra are plotted as a function of misorientation in figure 1 and the possible discrete misorientations are indicated for $p=60$. The ratio between the maximum and minimum grain boundary energy are $\sigma_{\max } / \sigma_{\min }=3.03$ for $\Delta \theta=1.5^{\circ}$ and $\sigma_{\max } / \sigma_{\min }=1.92$ for $\Delta \theta=3^{\circ}$. The grain boundary mobility $\mu_{g b}$ is assumed to be constant and equal to $1 * 10^{-6} \mathrm{~m}^{2} \mathrm{~s} / \mathrm{kg}$. The grain boundary width $\ell_{i, j}$ is taken $0.66 * 10^{-6} \mathrm{~m}$ for all misorientations. 


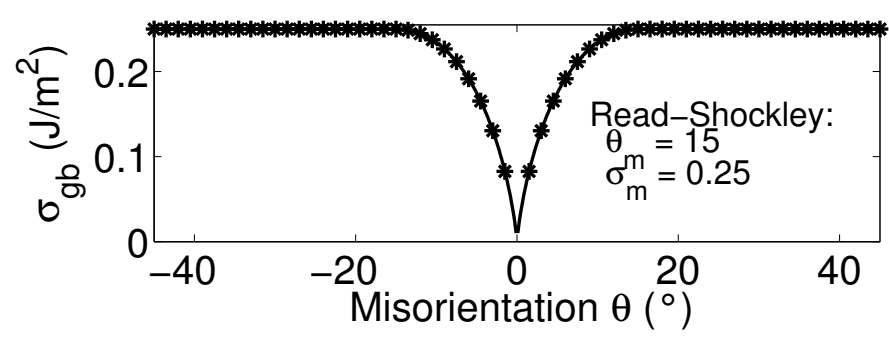

(a)

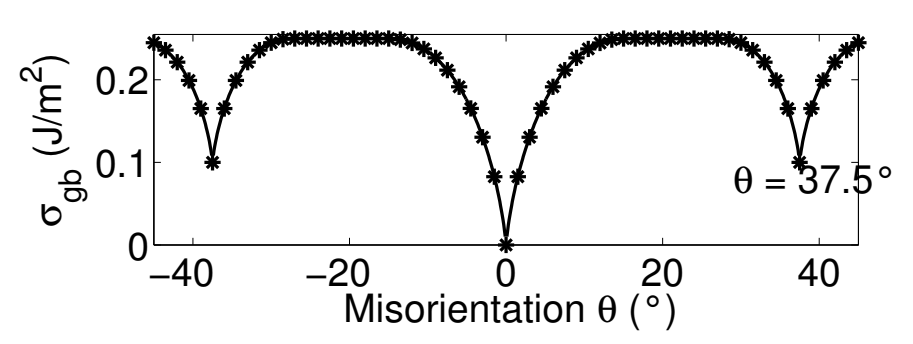

(b)

Figure 1. a) Type I and b) Type II of the misorientation dependent grain boundary energy used in this study.

The model parameters $\kappa_{i, j}, \gamma_{i, j}, m$ and $L$ that reproduce the desired grain boundary properties were calculated using the iterative algorithm described in [44], giving $m=2.25 * 10^{6} \mathrm{~J} / \mathrm{m}^{3}, L=2 \mathrm{~m} \cdot \mathrm{s} / \mathrm{kg}$ and values for $\kappa_{i, j}$ and $\gamma_{i, j}$ as shown in figure 2 for the energy of Type II and 60 possible orientations. For the energy of type I, $\kappa_{i, j}$ and $\gamma_{i, j}$ have the same values for the low misorientations, but equal $\kappa_{\max }$ and $\gamma_{\max }$ for all high-angle misorientations. If only 30 possible orientations are considered, only the values for $\theta=3,6,9^{\circ}, \ldots$ are used.

A standard explicit finite difference discretization with grid spacing $\Delta x=0.1 *$ $10^{-6} \mathrm{~m}$ and time step $\Delta t=0.008 \mathrm{~s}$ was used for the numerical solution of the phase field equation. Since grains are columnar and the grain orientations show axi-symmetry, simulations are performed in 2 D. The system size was $1024 \times 1024$ grid points $\left(102.4 \mu \mathrm{m}^{2}\right)$ and $500 \times 500$ grid points $\left(50.0 \mu \mathrm{m}^{2}\right)$ grid points.

The initial grain structures were generated as described by Fan and Chen [46]. First, small random values between -0.001 and 0.001 are assigned to all field vari- 
ables at all (discrete) positions in the system. Then, the neld variables are let to evolve according to equations (6) and assuming isotropic grain boundary properties until a polycrystalline microstructure is obtained. In this way, the initial grain orientations and grain boundary misorientations are random.

\subsection{Expected accuracy for grain boundary movement and triple junction angles}

For these model and simulation parameters, the movement of individual boundaries is reproduced with an accuracy of approximately $1.5 \%$ [44]. The cosines of most triple junction angles are reproduced with the same accuracy (We prefer to express the accuracy of triple junction angles as a function of the cosine of the angles, since the curvature of the boundaries and the accuracy depend linearly on this measure.) Triple junctions with angles far from $120^{\circ}$, namely those with an angle outside the range $\left[102^{\circ} 138^{\circ}\right]$, deviate more. In our simulations, this is the case if 2 boundaries with $\theta=1.5^{\circ}$ meet a boundary with $\theta=3^{\circ}$ or 2 boundaries with $\theta=3^{\circ}$ meet one with $\theta=1.5^{\circ}$. Another case is where a boundary with $\theta=1.5^{\circ}, 3^{\circ}$ or $37.5^{\circ}$ (Type II) meets 2 boundaries with maximum energy or 1 boundary with maximum energy meets two boundaries with $\theta=3^{\circ}$. Triple junctions where 2 boundaries with $\theta=1.5^{\circ}$ or $\theta=37.5^{\circ}$ (Type II) meet one with maximum energy are unstable (this is correctly reproduced in the simulations). Deviations can be of the order of $10 \%$ for the largest ratio of $\sigma_{\max } / \sigma_{\min }$ in the simulations with 60 possible orientations (i.e. $\left.\sigma_{\max } / \sigma_{\min }=0.25 / 0.0826\right)$. Accurate reproduction of these angles requires a smaller grid spacing $\Delta x$ or larger grain size. Moreover, the converence is slow for angles far from $120^{\circ}$.

As a consequence, it is not beneficial to increase further the number of possible orientations in our simulations without decreasing the grid spacing accordingly. If 
tation that can be represented is $\theta=0.75^{\circ}$ with, for the considered Read-Shockley

dependence (1) with $\theta_{m}=15^{\circ}$, a grain boundary energy $\sigma_{g b}=0.05 \mathrm{~J} / \mathrm{m}^{2}$ and hence $\sigma_{\max } / \sigma_{\min }=0.25 / 0.05=5$. It requires an even smaller grid spacing to reproduce these triple junctions well. Doubling the number of possible orientations would not increase the computational cost much; decreasing the grid spacing (and accordingly the time step) or increasing the system size, however, does considerably increase computer requirements. Since the purpose is to derive statistical information from the simulations, we have to consider a large number of grains and cannot take the grid spacing very small.

The difficulties with triple junction angles outside the range $\left[102^{\circ} 138^{\circ}\right]$ is due to the discrete nature and anisotropy of the discretization grid used in numerical simulations. Similar problems are experienced with other mesoscale simulation techniques [45]. In previous simulation studies, $\theta_{m}$ in the Read-Shockley relation, was sometimes taken larger than $15^{\circ}[18,23,35]$. Then, the misorientation dependence of the grain boundary energy for low misorientations is less steep and it might be possible to apply a finer resolution for the misorientation than in the present study for the same spatial resolution, but there is a limit as well. An advantage of phase-field models is that the range of easy-to-resolve triple junction angles can be modified to a certain extent (for a fixed resolution and grain size) by changing the width of the diffuse grain boundary profiles [44].

\section{Phase-field simulation results}

\subsection{Microstructures}

In figure 3 simulation images at different time steps are shown for a system with energy anisotropy of Type I. In figure 4 images are shown for a system with en- 


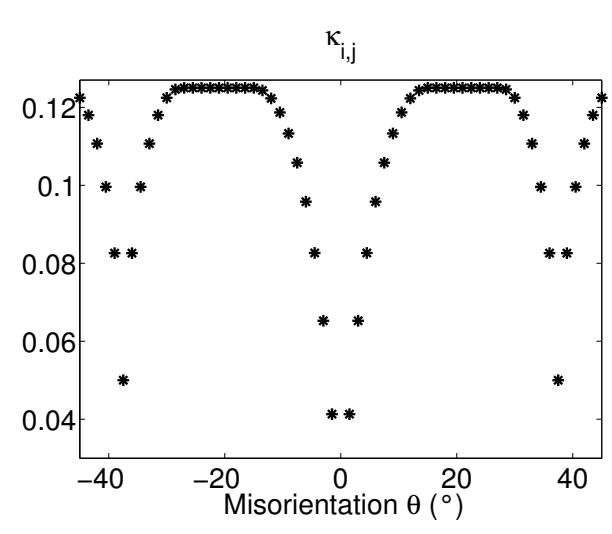

(a)

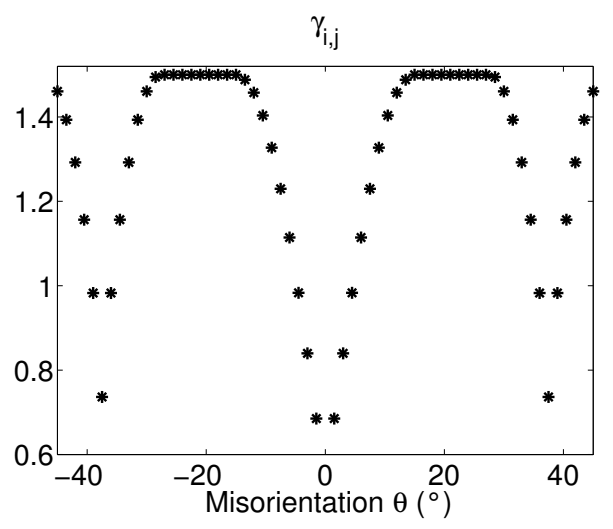

(b)

Figure 2. Phase field model parameters a) $\kappa_{i, j}$ and b) $\gamma_{i, j}$ as a function of misorientation for an energy of type II. $\kappa_{i, j}$ ranges between 0.0413 and 0.125 and $\gamma_{i, j}$ between 0.685 and 1.5 .

ergy anisotropy of Type II. Boundaries with high energy are plotted with darker and thicker lines than boundaries with low energy. The high-energy boundaries clearly dominate the evolution of the structure. Except for a few dangling boundaries, they form an individual grain boundary network with almost uniform grain boundary properties and triple junction angles of $120^{\circ}$. The low-energy boundaries are located within the grains formed by the high-energy grain boundary network. Most often they cross the grains from one side to the opposite. At later stages in the simulations, the low-energy boundaries can also form sub-networks within the grains of the high-energy boundaries. Due to their lower energy, these networks coarsen much slower than the high-energy boundary network. In fact, the lowenergy boundaries mainly follow the movement of the high angle boundaries. They lengthen when located in a growing grain and shorten when located in a shrinking grain. Because of the large difference in grain boundary energy, the low-energy boundaries intersect the high-energy boundaries almost perpendicularly so that they hardly affect the configuration of the high-energy boundary network. Both systems show similar grain growth behavior. We do not observe a large amount of higher-order junctions and they are not stable. The average number of faces is 


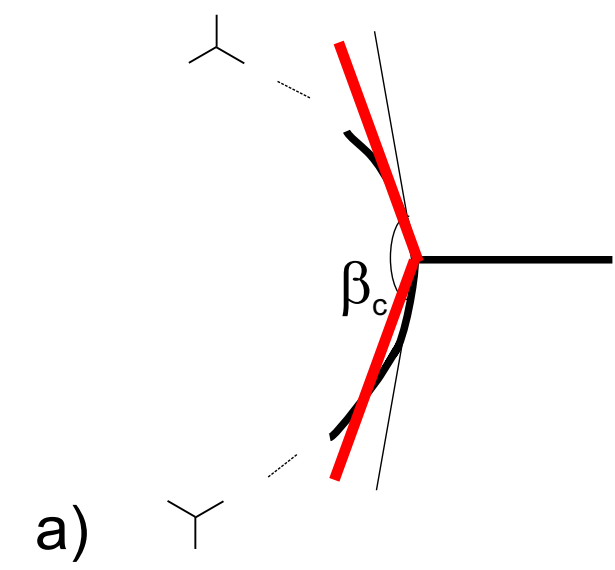

b)

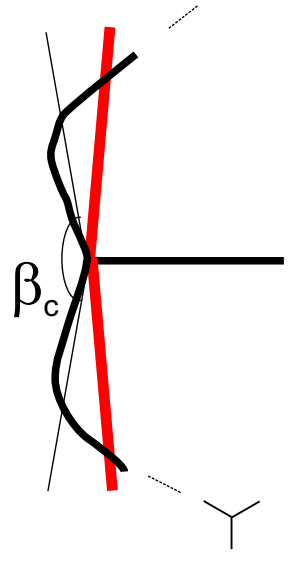

Figure 5. Schematic representation of triple junctions formed by 2 high-energy boundaries $\left(\sigma_{g b}=0.25 \mathrm{~J} / \mathrm{m}^{2}\right)$ and 1 boundary with energy $\sigma_{g b}=0.0826 \mathrm{~J} / \mathrm{m}^{2}\left(\theta=1.5^{\circ}\right)$ for the cases where the high-energy boundaries are curved a) away or b) towards the low-energy boundary. The red line gives the direction of the boundary obtained with low spatial resolution.

a)

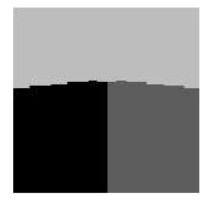

b)

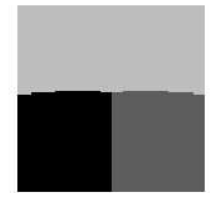

c)

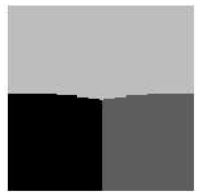

d)

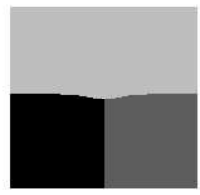

e)

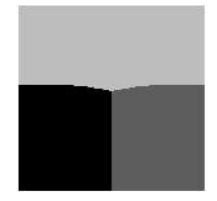

Figure 6. Simulation images obtained for increasing numerical accuracy for an isolated moving triple junction with $\sigma_{g b}=0.25 \mathrm{~J} / \mathrm{m}^{2}$ for the horizontal boundaries and $\sigma_{g b}=0.0826 \mathrm{~J} / \mathrm{m}^{2}$ for the vertical boundary. a) $\Delta x=0.1, \ell_{i, j}=0.66$, b) $\Delta x=0.1, \ell_{i, j}=1.32$, c) $\Delta x=0.1$, $\ell_{i, j}=2.64$, d) $\Delta x=0.05, \ell_{i, j}=2.64$ and e) $\Delta x=0.025, \ell_{i, j}=2.64$.

junctions formed by a boundary with an energy equal or lower than $\sigma_{g b}=0.0826$ $\mathrm{J} / \mathrm{m}^{2}$ and 2 high-energy boundaries all give triple junction angles within the same range of values. The exact value rather depends on the local geometry than on the energy of the boundaries.

\subsection{Grain growth kinetics}

In figure 7 , the evolution of the mean grain size as a function of time is plotted for a system with energy anisotropy of type II and using 60 possible discrete orientations. The effective mean grain area follows a power growth law with $n_{\text {eff }}$ close to 1 , after a short transition time. Since the simulation images in figures 3 and 4 show that the high-energy boundaries form an almost independent grain boundary network, the mean grain size of the grain structures formed by all boundaries with an energy 


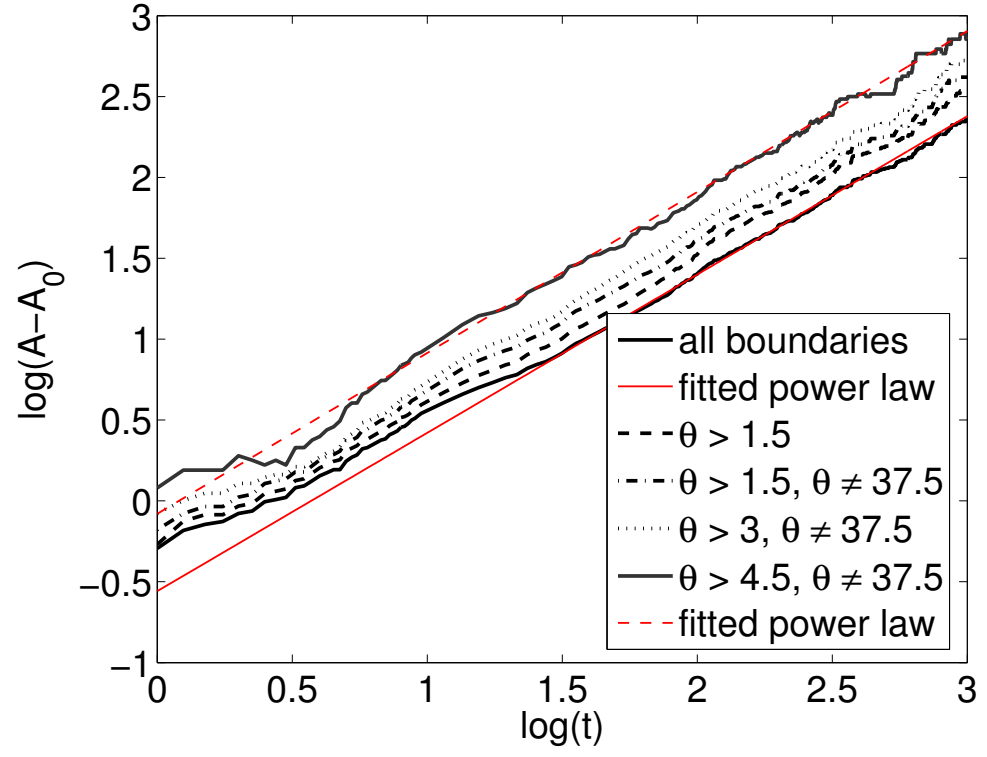

Figure 7. Evolution of the mean grain area as a function of time obtained from a simulation with an energy misorientation dependence of type II, 60 possible orientations and a system size of $1024 \times 1024$ grid points. The evolution of the mean grain area of different superstructures formed by all grain boundaries with an energy higher than a certain threshold value is also plotted. It is indicated in the legend which boundaries are included in the network for the different curves. For two curves, the data are fitted to the power growth law (2), giving $n_{\text {eff }}=1.00$ for the complete boundary network and $n_{h}=1.02$ for a network consisting of only the high-energy boundaries.

higher than a certain threshold value, was determined as well. The curves for these superstructures (most of the grains formed by these networks contain multiple grains of the actual grain boundary network) are also plotted in figure 7 . As the grain boundary energy of these networks is more homogeneous, it is not surprising that fitting of the data points to the power growth law (2) gives a value for $n_{\theta \text {-cutoff }}$ close to 1 . A growth exponent close to 1 was obtained for all systems of $1024 \times 1024$ grid points. The systems of $500 \times 500$ grid points do not contain enough grains to reach the regime where $n_{e f f}=1$, although some of the superstructures can.

\subsection{Grain boundary misorientation distribution (MDF)}

In figures $8 \mathrm{a}$ and $\mathrm{b}$, the number and length weighted MDF are plotted as a function of absolute misorientation at different times, for a system with grain boundary energy of type II and 60 possible orientations. They are uniform for the initial microstructure. During grain growth, the number and length fraction of boundaries 
than for the number weighted, probably because of geometric lengthening of the low-energy boundaries [18]. After a finite amount of time, the misorientation distributions no longer change, except for statistical variations. The shape of the final MDF's correlates with the misorientation dependence of the grain boundary energy. The final number and length fraction (i.e. number or length density $\times \Delta \theta$ ) for the misorientation with lowest energy $\left(\theta=1.5^{\circ}\right)$ are approximately 0.11 and 0.26 , and for that with second lowest energy $\left(\theta=37.5^{\circ}\right)$ are approximately 0.06 and 0.1 . All other misorientations, namely $3^{\circ} \leq \theta \leq 36^{\circ}$ or $39^{\circ} \leq \theta \leq 45^{\circ}$, have almost the same number and length fraction, within the statistical variations. To illustrate this behavior more clearly, the number and length fraction of special boundaries is plotted as a function of time in the figures $8 \mathrm{c}$ and $\mathrm{d}$. They evolve towards a constant value, which is higher for boundaries with a lower energy.

In figure 9 , the same quantities are plotted for a system with the same grain boundary energy (Type II), but using 30 possible orientations. Also in this case the number and length weighted MDF's become non-uniform with higher possibilities for boundaries with lower energy and are constant in time after a finite amount of grain growth. The final number and length fraction for the misorientation with lowest energy $\left(\theta=3^{\circ}\right)$ are approximately 0.16 and 0.27 . The number and length fraction of boundaries with misorientation $\theta=36^{\circ}$ or $\theta=39^{\circ}$ increased slightly to a value 0.09 and 0.1 , respectively. All other misorientations have almost equal number and length fractions. From a quantitative point of view, these final fractions of lowangle and special high-angle boundaries obtained for $\Delta \theta=3^{\circ}$ (30 orientations) are very different from those obtained for $\Delta \theta=1.5^{\circ}$ (60 orientations).

Figure 10 compares the final length weighted MDF's obtained using 30 and 60 discrete orientations. Qualitatively, they have a similar shape: both have the highest 
peak at the smallest misorientation and another peak around the special highangle misorientation. The peaks are however higher for the case with 60 discrete orientations. This set namely contains the misorientations $\theta=1.5$ and $\theta=37.5$ with considerably lower energies, which are not present in the set of 30 discrete misorientations. If, for example, 120 orientations would have been considered ( $\Delta \theta=$ 0.75 ), this set would contain the misorientation $\theta=0.75$ (not present in the set with 30 or 60 orientations) with an energy $\sigma_{g b}=0.05$ which is considerably lower than the energy for the other misorientations. This will affect the heights and shapes of the two peaks in the MDF's. Because of the logarithmic term in the Read-Shockley equation for grain boundary energy, the height of the peaks is thus quite sensitive to the number of possible discrete misorientations. The grain boundary energy namely decreases fast for $\theta \rightarrow 0$ or $\theta \rightarrow \theta_{\text {cusp. }}$. A very small discretization with respect to orientation (which requires a very fine spatial and temporal resolution as described in section 2.3) is probably required to minimize this dependence on $\Delta \theta$.

For an energy of type I, the MDF's have a single peak at the smallest misorientation. The final number and length fraction of the boundary with lowest energy are 0.12 and 0.27 when 60 orientations are considered $\left(\theta=1.5^{\circ}\right)$, and approximately 0.17 and 0.3 when 30 orientations $\left(\theta=3^{\circ}\right)$. The fraction of low-angle boundaries is thus slightly higher in the absence of energy cusps at high misorientations.

Figure 11 shows that, although the misorientation distribution changes shape, the distribution of grain orientations remains uniform during grain growth. Only after a considerable amount of grain growth, some of the grain orientations vanish as the number of grains decreases in time. 


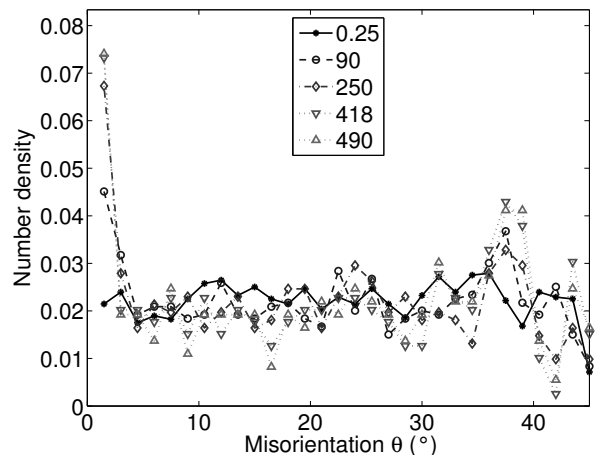

(a)

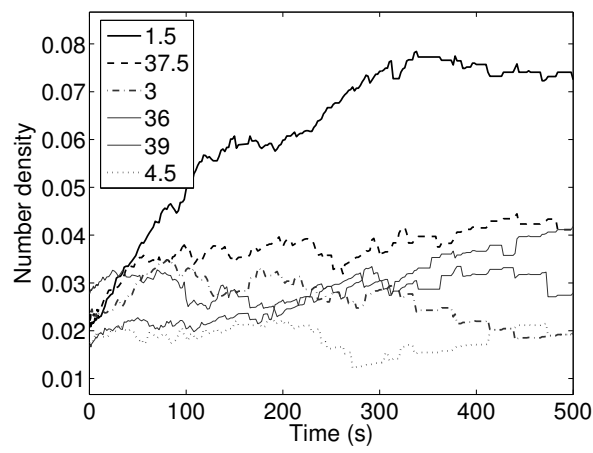

(c)

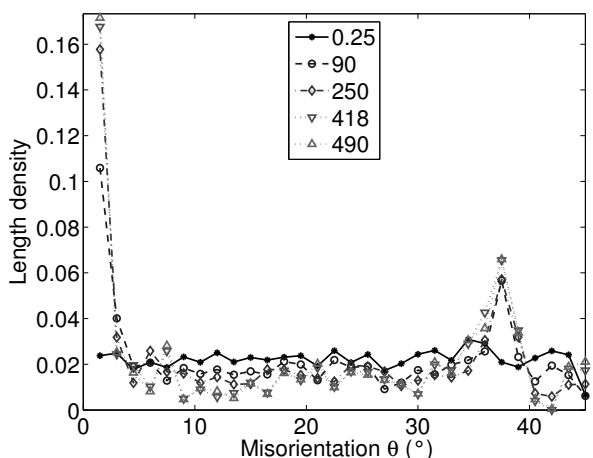

(b)

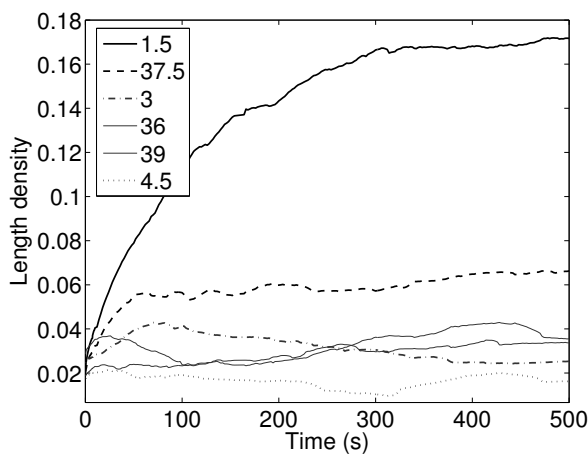

(d)

Figure 8. (a) Number and (b) length weighted MDF at different times and temporal evolution of the (c) number and (d) length fraction of special boundaries for a system with anisotropic grain boundary energy of Type II, 60 possible orientations and a system size of $1024 \times 1024$ grid points.

\section{Mean field analysis}

In this section a mean field approach, starting from the parabolic growth law obtained for steady-state grain growth in isotropic materials [15, 47-49], is performed to calculate the growth exponent for structures with non-uniform grain boundary energy.

The images in figures 3 and 4 reveal that the high-energy boundaries form an independent grain boundary network with almost uniform grain boundary energy. This network can be assumed to form a grain structure of $N$ grains with isotropic grain growth behavior. The average grain area of the high-energy boundary network 


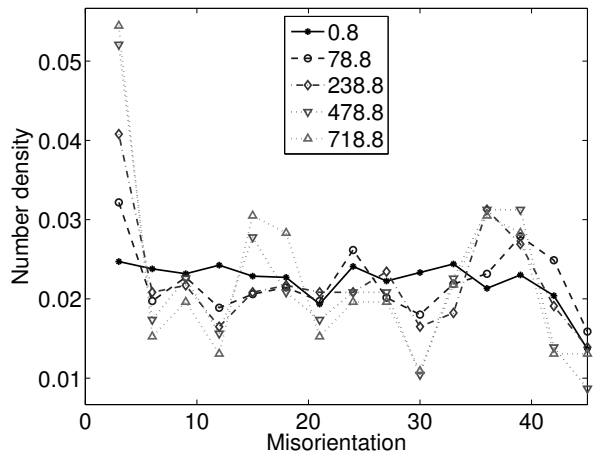

(a)

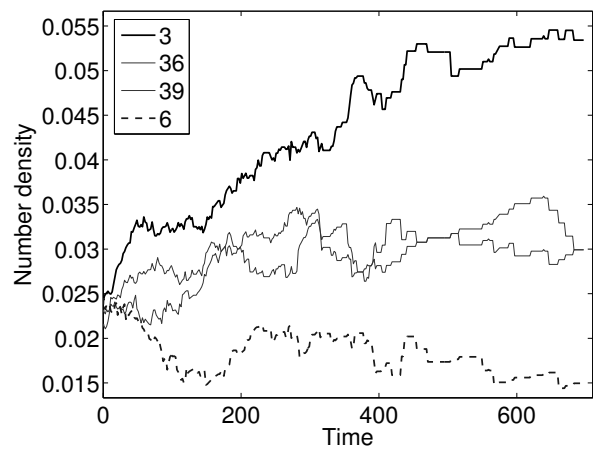

(c)

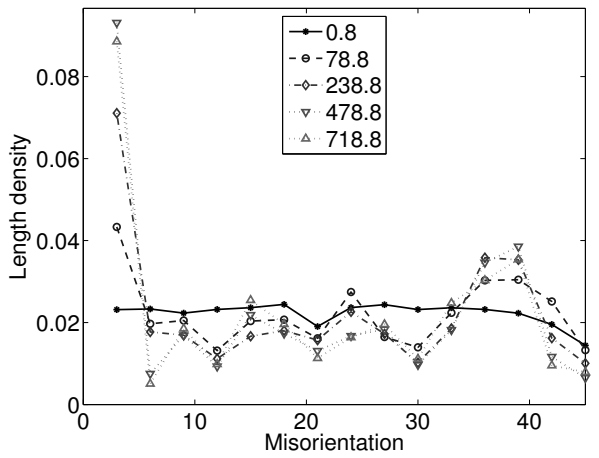

(b)

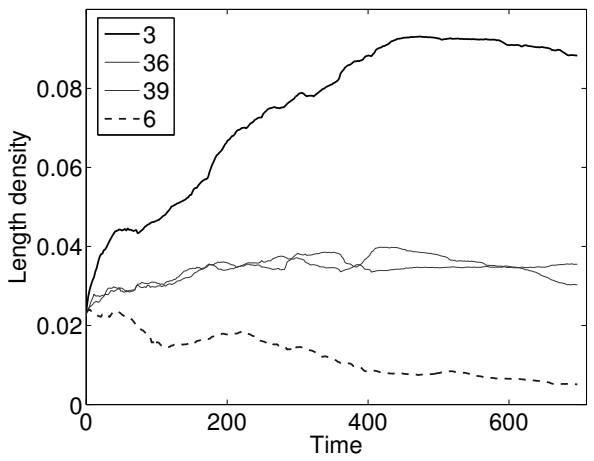

(d)

Figure 9. (a) Number and (b) length weighted MDF at different times and temporal evolution of the (c) number and (d) length fraction of special boundaries for a system with anisotropic grain boundary energy of Type II, 30 possible orientations and a system size of $1024 \times 1024$ grid points.

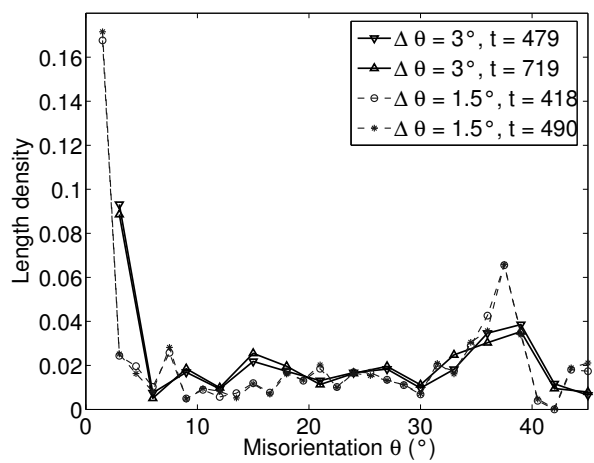

Figure 10. Comparison of the final length weighted MDF's obtained from simulations for systems with anisotropic grain boundary energy of Type II using 30 and 60 discrete orientations.

$A_{h}$ can accordingly be written as $[15,47-49]$

$$
A_{h}=\frac{A_{t o t}}{N},
$$




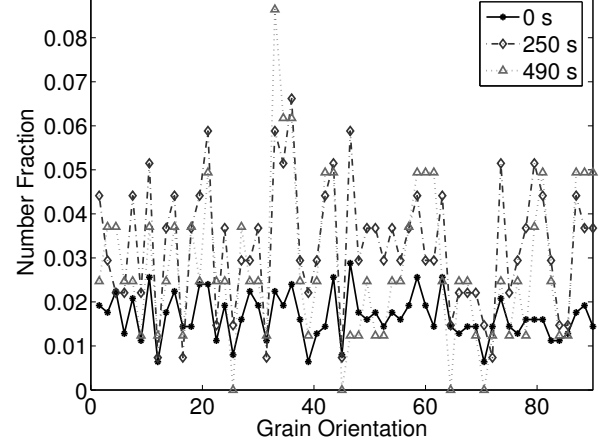

Figure 11. Orientation distribution at different times for a system with energy of type II, 60 possible orientations and a system size of $1024 \times 1024$ grid points.

with $A_{t o t}$ the total area of the system. The evolution of the mean grain area $A_{h}$ follows a parabolic growth law

$$
A_{h}=A_{h}(0)+k t
$$

with $t$ time, $k$ a kinetic constant related to the grain boundary energy and mobility and $A_{h}(0)$ the average grain area at time $t=0$.

Next, based on the simulation images, we assume that each low-energy boundary in the anisotropic system increases the effective number of grains in the system by 1. With $N^{\prime}$ the number of boundaries with low energy, the effective mean grain area of the grains formed by the anisotropic grain boundary network can then be written as

$$
A_{e f f}=\frac{A_{t o t}}{N+N^{\prime}}
$$

or using (12) and (13)

$$
A_{e f f}=\frac{A_{t o t}}{\frac{A_{t o t}}{A_{h}(0)+k t}+N^{\prime}}=\frac{1}{\frac{1}{A_{h}(0)+k t}+\frac{N^{\prime}}{A_{t o t}}}=\frac{A_{h}(0)+k t}{1+\frac{N^{\prime}}{N}} .
$$




$$
\begin{aligned}
\log \left(A_{e f f}\right) & =-\log \left(\frac{1}{A_{h}(0)+k t}+\frac{N^{\prime}}{A_{t o t}}\right) \\
& =\log \left(A_{h}(0)+k t\right)-\log \left(1+\frac{N^{\prime}}{N}\right) .
\end{aligned}
$$

The effective growth exponent $n_{\text {eff }}$ for the anisotropic grain structure is accordingly calculated as

$$
\begin{aligned}
n_{e f f} & =\frac{\mathrm{d} \log \left(A_{e f f}\right)}{\mathrm{d} \log (t)}=\frac{\mathrm{d} \log \left(A_{e f f}\right)}{\mathrm{d} t} t \\
& =-\frac{t}{\frac{1}{A_{h}(0)+k t}+\frac{N^{\prime}}{A_{t o t}}}\left[\frac{-k}{\left(A_{h}(0)+k t\right)^{2}}+\frac{1}{A_{t o t}} \frac{\mathrm{d} N^{\prime}}{\mathrm{d} t}\right] \\
& =\frac{k t}{A_{h}(0)+k t}-\frac{t}{1+\frac{N^{\prime}}{N}} \frac{\mathrm{d}\left(N^{\prime} / N\right)}{\mathrm{d} t},
\end{aligned}
$$

where it is applied that for $t \rightarrow \infty, A_{e f f}-A_{0, e f f}=k_{e f f} t^{n_{e f f}}$ goes asymptotically to $A_{e f f}=k_{e f f} t^{n_{e f f}}$.

Below, equations (18)-(19) are interpreted assuming different limiting situations

(1) If there are no low-energy boundaries in the system $\left(N^{\prime}=0\right)$, equation (18) reduces to

$$
n_{e f f}=k t /\left(A_{h}(0)+k t\right)
$$

which goes to 1 for $t$ large. Furthermore,

$$
\frac{\mathrm{d} A_{e f f}}{\mathrm{~d} t}=k=k_{e f f},
$$

as expected for normal grain growth in an isotropic system. 
1, the number of high-energy boundaries decreases much faster than the number of low-energy boundaries. In this case, the number of low-energy boundaries $N^{\prime}$ can be considered to be constant within limited time intervals. Such a condition is approximately obeyed at the beginning of the simulations. Equation (18) then gives

$$
n_{e f f}=\frac{k t}{A_{h}(0)+k t} \frac{1}{1+\frac{N^{\prime}}{N}}
$$

and for $t$ large

$$
n_{e f f}=\frac{1}{1+\frac{N^{\prime}}{N}}
$$

The growth exponent is thus smaller than 1 and changes in time.

(3) In our simulations, the system evolves towards a regime where the MDF's are nearly constant in time. Since the average number of sides of a grain in the isotropic high-energy boundary network is 6 , a constant number fraction of low energy boundaries involves a constant ratio $N^{\prime} / N$. For such conditions, the second term on the right hand side in relation (19) equals 0 . The effective growth exponent $n_{\text {eff }}$ thus equals

$$
n_{e f f}=\frac{k t}{A_{h}(0)+k t},
$$

which goes to 1 for large $t$. This is consistent with the simulation data presented in figure 7 . Moreover, taking the derivative with respect to time from equation (15) 


$$
\frac{\mathrm{d} A_{e f f}}{\mathrm{~d} t}=\frac{k}{1+\frac{N^{\prime}}{N}}=k_{e f f}
$$

It follows from equation (25) that the prefactor $k_{\text {eff }}$ in the growth law is thus lowered by the presence of low-energy boundaries, which is also in agreement with the curves plotted for different superstructures in 7 . This situation is thus most relevant for the present study.

One might wonder whether and under which conditions it is reasonable to assume that $N^{\prime} / N$ remains constant during grain growth. There are many events by which the number of grain boundaries and grains in the system changes [15, 26]. One event is the disappearance of a grain from the high-energy grain boundary network. This is always associated with the annihilation of all low-energy boundaries inside this grain. Such a process does therefore not change the ratio $N^{\prime} / N$. Furthermore, neighbor switching events in the high-energy grain boundary network do not remove a grain, but may create a low-energy boundary. They can accordingly increase the ratio $N^{\prime} / N$. Since the grain orientations in the plane of the film are random for fiber textures, the possibility to create a new low-energy boundary is proportional to the fraction of misorientations with a low energy. Besides the coarsening of the highenergy network, the low-energy substructures coarsen as well, although on a longer time scale. This results in a decrease of the number of low-energy boundaries, but does not affect the number of grains in the high-energy grain boundary network. Coarsening of the low-energy substructures therefore lowers the ratio $N^{\prime} / N$. The frequency with which low-energy boundaries are annihilated with respect to the frequency of the 2 other events, depends on the number fraction of low-energy boundaries and the difference in velocity between low- and high-energy boundaries. 
In order to maintain a steady-state regime with $N / N$ constant and $n=1$, the two latter processes must balance each other on average. This seems to be the case for the systems considered in the present study and for 3D structures with fully random texture [18]. It is, for example, not the case for structures with one or a few strong texture components, which rather undergo texture strengthening [18, 20], a change in texture $[22,25,30-32]$ or abnormal grain growth [21].

We believe that such a regime with $N^{\prime} / N$ constant and $n=1$ can always be established after a finite transition period, in fiber textured systems with realistic grain boundary energy and mobility. The main reason is the random distribution of grain orientations in the plane of the film. The possibility to form low-energy or special boundaries is accordingly relatively small compared to that to form a high-energy boundary, even if more low-energy cusps are considered. The fractions of boundaries with low energy are higher than those with high energy because low-energy boundaries remain in the system for a longer time. They can however not remain for infinite time: as the low-energy boundaries form networks within the grains of the high-energy boundary network, low-energy boundaries are disappearing continuously together with shrinking grains of the high-energy boundary network. The final MDF for which there is a balance between all processes described in the above section depends (in a complex way) on the relative velocities of the different boundaries, the possibility to form low-energy boundaries of a certain type (i.e. the number and width of the cusps) and on the triple junction geometries, which are all related to the misorientation dependence of the grain boundary energy. The exact correlation between the misorientation dependence of the grain boundary energy and the final MDF is currently not known.

Furthermore, we do not expect that mobility anisotropy will prevent a fiber textured system from finding a regime with constant MDF. The mean field study 
and grain size distribution of fiber textured systems with uniform grain boundary energy. Therefore, in the presence of mobility anisotropy, it can still be assumed that the growth exponent of the high-energy boundary network equals 1 so that equation (13) and our mean field analysis remain valid. The rate at which lowenergy boundaries are created and annihilated can however be affected by the mobility anisotropy, and the ratio $N^{\prime} / N$, or final MDF, can accordingly be different. For example, if the low-energy boundaries have a lower mobility, $N^{\prime} / N$ must be higher; since the coarsening of the low-energy networks is slower, it must be denser so that the number of vanishing grain boundaries per unit of time equals the rate at which low-energy boundaries are created by neighbor switching events in the high-energy boundary network. If the low energy boundaries have higher mobility, $N^{\prime} / N$ must be lower.

If the initial grain orientations are not random, on the other hand, certain types of boundaries have a higher possibility to form when a grain disappears or from a neighbor switching event. As a consequence, the evolution of the MDF is governed by both, the misorientation dependence of the grain boundary energy (and mobility) and the initial grain orientation distribution. Then, it might require extensive grain growth or even be impossible to establish a steady-state regime where there is an equilibrium between the annihilation and formation of boundaries for each misorientation, for example in the extreme cases where all orientations are around one or a few texture components or with just a few randomly oriented grains for which a continuous change in texture is observed [18, 20-22, 25, 30-32]. It is not clear how large the deviations from a random orientation distribution must be to induce phenomena such as texture strengthening and abnormal grain growth.

From the previous analysis we conclude that if a growth exponent $n<1$ was 
obtained trom previous grain growth simulations tor tiber textured systems, this is

highly probably because the system size is too small, the simulation times are too short or because the initial grain orientations were not fully random.

\section{Conclusions}

Despite quite a number of studies on anisotropic grain growth, there is no systematic understanding yet of how the characteristics of the grain structure and grain boundary network evolve in time for anisotropic materials with a fiber texture. We performed grain growth simulations using a generalized phase field model for columnar grain structures with a fiber texture and misorientation dependent grain boundary energy. The phase-field model allows accurate quantification of the grain boundary energy for each grain boundary individually as a function of its misorientation. Two types of misorientation dependence of the grain boundary energy were assumed; one with a Read-Shockley dependence for low misorientations $\left(\theta<15^{\circ}\right)$ and constant energy at high misorientations and another with an extra low-energy cusp around a misorientation of $37.5^{\circ}$. The energy anisotropy is assumed to have 4-fold symmetry. The initial grain structures have random planar orientations.

In all cases, grain boundaries with high energy form an independent network that drives the grain growth behavior. Boundaries with low energy are located within the grains formed by this high-energy boundary network and follow the movement of the high-energy boundaries. If the system size is large enough, a steady-state regime is reached after a finite amount of grain growth where the MDF's are constant in time and the mean grain area evolves according to a power growth law with $n_{\text {eff }}$ close to 1 . Qualitatively, we can conclude that both the number and length weighted MDF are correlated with the grain boundary energy. Grain boundaries with lower energy have a higher number and length fraction. The 
when in contact with boundaries with a higher energy to fulfill the equilibrium requirements at the triple junctions. Quantitatively, the grain boundary fractions obtained for different misorientations are quite sensitive to the number of discrete orientations considered in the simulation. The reason is that, due to the logarithmic Read-Shockley dependence of the grain boundary energy at low misorientations and near cusps, the minimum energy values considered in simulations with discrete orientations largely decrease when smaller misorientations are considered.

A mean field approach, starting from the parabolic growth law for normal grain growth in isotropic materials, shows that it is very probable to obtain a steadystate regime with $n=1$ in fiber textured systems with non-uniform grain boundary properties.

Important challenges for future work are an accurate representation of triple junction angles in mesoscale grain growth simulations and the derivation of an exact relation between grain boundary energy, grain boundary mobility and the final MDF, as it is actually the fraction and spatial distribution of the low-angle and special boundaries that plays an important role in many practical applications.

\section{References}

[1] C.V. Thompson, Ann. Rev. Mater. Sci. 30 (2000) 159.

[2] S. Vaidya and A.K. Sinha, Thin solid films 75 (1981) 253.

[3] D.B. Knorr and K.P. Rodbell, J. Appl. Phys. 79 (1996) 2409.

[4] A. Gungor, K. Barmak, A.D. Rollett, C. Cabral Jr. and J.M. E. Harper, J. Vac. Sci. Technology B $20(2002) 2314$.

[5] G. Palumbo, E.M. Lehockey and P. Lin, JOM 50 (1998) 40.

[6] W.T. Read and W. Shockley, Phys. Rev. B 78 (1950) 275.

[7] A.P. Sutton and R.W. Balluffi, Acta Metall. 35 (1987) 2177.

[8] D. Wolf, Acta Metall. 37 (1989) 1983.

[9] D.G. Brandon, Acta Metall. 14 (1966) 1479. 
[10] M. Furtkamp, G. Gottstein, D.A. Molodov, V.N. Semenov and L.S. Shvindlerman, Acta Metall. 46

(1998) 4103.

[11] H.J. Frost, F. Spaepen and M.F. Ashby, Scripta Metall. 16 (1982) 1165.

[12] S.M. Foiles and J.J. Hoyt, Acta Mater. 54 (2006) 3351.

[13] D.M. Saylor, B.S. El Dasher, A.D. Rollett and G.S. Rohrer, Acta Mater. 52 (2004) 3649.

[14] W.E. King, Scripta Mater. 38 (1998) 449.

[15] C.V. Thompson, Solid State Physics (Academic Press) 55 (2000) 269.

[16] T. Watanabe, Scripta Metall. Mater. 27 (1992) 1497.

[17] J. Gruber, D.C. George, A.P. Kuprat, G.S. Rohrer and A.D. Rollett, Scripta Mater. 53 (2005) 351.

[18] E.A. Holm, G.N. Hassold and M.A. Miodownik, Acta Mater. 49 (2001) 2981.

[19] G.N. Hassold, E.A. Holm and M.A. Miodownik, Mater. Sci. Technol. 19 (2003) 683.

[20] A. Kazaryan, Y. Wang, S.A. Dregia and B.R. Patton, Acta Mater. 50 (2002) 2491.

[21] A.D. Rollett, D.J. Srolovitz and M.P. Anderson, Acta Metall. 37 (1989) p. 1227.

[22] N. Ma, A. Kazaryan, S.A. Dregia and Y. Wang, Acta Mater. 52 (2004) 3869.

[23] G.S. Grest, D.J. Srolovitz and M.P. Anderson, Acta Metall. 33 (1985) 509.

[24] M. Upmanyu, G.N. Hassold, A. Kazaryan, E.A. Holm, Y. Wang, B. Patton and D.J. Srolovitz, Interface Science 10 (2002) p. 202.

[25] O.M. Ivasishin and S.V. Shevchenko, N.L. Vasiliev and S.L. Semiatin, Acta Mater. 51 (2003) 1019.

[26] C.S. Smith, Metal Interfaces (Am. Soc.Metals, Cleveland) (1952) 65.

[27] N. Ono, K. Kimura and T. Watanabe, Acta Mater. 47 (1999) 1007.

[28] N.M. Hwang, J. Mater. Sci. 33 (1998) 5625.

[29] N.M. Hwang, S.B. Lee and D.-Y. Kim, Scripta Mater. 44 (2001) 1153.

[30] K. Mehnert and P. Klimanek, Comp. Mater. Sci. 9 (1997) 261.

[31] H. Eichelkraut, G. Abbruzzese and K. Lücke K., Acta Metall. 36 (1988) 55.

[32] N.M. Hwang, B.-J. Lee and C.H. Han, Scripta Mater. 37 (1997) 1761.

[33] N.M. Hwang, Scripta Mater. 37 (1997) 1637.

[34] A. Kazaryan, B.R. Patton, S.A. Dregia and Y. Wang, Acta Mater. 50 (2002) 499.

[35] Q. Yu and S.K. Esche, Mater. Lett. 56 (2002) 47.

[36] Y. Saito and M. Enomoto, ISIJ Int. 32 (1992) 267.

[37] H.J. Frost, Y. Hayashi, C.V. Thompson and D.T. Walton, Materials Research Society Symposium Proceedings, 338 (1994) 295.

[38] H.J. Frost and C.V. Thompson, Current opinion in solid state \& materials science 1 (1996) 361.

[39] W.J. Boettinger, J.A. Warren, C. Beckermann and A. Karma, Annu. Rev. Mater. Res. 32 (2002) 163.

[40] L.-Q. Chen, Annu. Rev. Mater. Res. 32 (2002) 113.

[41] I. Singer-Loginova and H.M. Singer, Rep. Prog. Phys. 71 (2008) 106501

[42] N. Moelans, B. Blanpain and P. Wollants, Computer Coupling of Phase Diagrams and Thermochemistry (CALPHAD) 32 (2008) 268.

[43] N. Moelans, B. Blanpain and P.Wollants, Phys. Rev. Lett. 101 (2008) 025502. 
[45] E.A. Holm, M.A. Miodownik and A.D. Rollett, Acta Mater. 51 (2003) 2701

[46] D. Fan and L.-Q. Chen, Acta Mater. 45 (1997) 611.

[47] J.E. Burke and D. Turnbull, Prog. Met. Phys. 3 (1952) 220.

[48] M.Hiller, Acta Metall. 13 (1965) 227.

[49] W.W. Mullis, Acta Mater. 46 (1998) 6219. 